\title{
Genomic Analysis of the Chicken Infectious Anemia Virus in a Specific Pathogen-Free Chicken Population in China
}

\author{
Yang Li, Yixin Wang, Lichun Fang, Jiayuan Fu, Shuai Cui, Yingjie Zhao, Zhizhong Cui, \\ Shuang Chang, and Peng Zhao
}

College of Veterinary Medicine, Shandong Agricultural University, Taian 271018, China

Correspondence should be addressed to Shuang Chang; changshuang81@126.com and Peng Zhao; zhaopeng@sdau.edu.cn

Received 11 January 2016; Revised 19 April 2016; Accepted 8 May 2016

Academic Editor: Kent M. Reed

Copyright (c) 2016 Yang Li et al. This is an open access article distributed under the Creative Commons Attribution License, which permits unrestricted use, distribution, and reproduction in any medium, provided the original work is properly cited.

\begin{abstract}
The antibody to chicken infectious anemia virus (CIAV) was positive in a specific pathogen-free (SPF) chicken population by ELISA test in our previous inspection, indicating a possible infection with CIAV. In this study, blood samples collected from the SPF chickens were used to isolate CIAV by inoculating into MSB1 cells and PCR amplification. A CIAV strain (SD1403) was isolated and successfully identified. Three overlapping genomic fragments were obtained by PCR amplification and sequencing. The full genome sequence of the SD1403 strain was obtained by aligning the sequences. The genome of the SD1403 strain was $2293 \mathrm{bp}$ with a nucleotide identity of $94.8 \%$ to $98.5 \%$ when compared with 30 referred CIAV strains. The viral proteins VP2 and VP3 were highly conserved, but VP1 was not relatively conserved. Both amino acids 139 and 144 of VP1 were glutamine, which was in accord with the low pathogenic characteristics. In this study, we first reported that CIAV exists in Chinese SPF chicken populations and may be an important reason why attenuated vaccine can be contaminated with CIAV.
\end{abstract}

\section{Introduction}

Chicken infectious anemia virus (CIAV) can cause the atrophy of bone marrow hematopoietic and lymphoid tissues (e.g., thymus) in young chickens, leading to aplastic anemia and immune suppression. The infection has been very common in chicken populations worldwide [1-4] since first reported CIAV in 1979 [5].

CIAV belongs to the Gyrovirus genus of the Circoviridae family, and the genome has three open reading frames (ORFs) which encode VP1, VP2, and VP3 [2]. VP1 and VP2 are protective antigen proteins which are encoded to produce neutralizing antibodies [6]. Except for VP1, the amino acid (AA) composition of CIAV was relatively conserved. Previous studies have indicated that AAs 139-151 are a highly variable region of the VP1 gene, and AA 139 or 144 could especially affect virus replication and cell infection [7]. Generally speaking, if both AA 139 and 144 in the loci are glutamines, replication and infective ability of the virus are relatively weaker [7].

It was reported that CIAV often contaminated attenuated vaccines to infect chicken populations $[4,8]$, and the use of CIAV-contaminated specific pathogen-free (SPF) chicken embryos is one of the important reasons for CIAV infection of avian attenuated vaccine. In the current study, a CIAV strain was isolated from a Chinese SPF chicken population, and the sequence of its full genome was analyzed.

\section{Materials and Methods}

2.1. Background of the SPF Chicken Population. According to guidelines, the detection of antibodies in SPF chicken flocks must be carried out regularly to evaluate the infection status of different pathogenic bacteria and viruses. CIAV antibody test kit (IDEXX, Beijing, China) was used to investigate the positive rate of CIAV antibody in one of SPF flocks in China. The positive rate of CIAV antibody in this flock was $20 \%$. Blood samples were collected from those chickens, and blood DNA was isolated using a DNA extraction kit (Omega, USA). Subsequently, PCR experiments were carried out according to the methods in previous studies to detect viral genome DNA of CIAV [8].

2.2. Isolation and Detection of Virus. Blood samples positive for CIAV antibody were collected from ten individual 
TABLE 1: Information of CIAV reference strains and amino acids in highly variable regions of VP1 protein.

\begin{tabular}{|c|c|c|c|c|c|}
\hline \multirow[t]{2}{*}{ Strains } & \multirow[t]{2}{*}{ Country and time } & \multirow[t]{2}{*}{ Accession number } & \multirow[t]{2}{*}{ Whole length } & \multicolumn{2}{|c|}{$\begin{array}{c}\text { Amino acid } \\
\text { position of VP1 }\end{array}$} \\
\hline & & & & 139\# & $144 \#$ \\
\hline M81223 & 1993, Germany & M81223 & $2298 \mathrm{bp}$ & Lysine & Aspartate \\
\hline CAU65414 & 1996, Australia & CAU65414 & $2298 \mathrm{bp}$ & Glutamine & Glutamine \\
\hline TR20 & 1999, Japan & AB027470 & $2298 \mathrm{bp}$ & Glutamine & Glutamine \\
\hline L14767 & 1999, USA & L14767 & $2298 \mathrm{bp}$ & Glutamine & Glutamine \\
\hline $\mathrm{A} 2$ & 2000, Japan & AB031296 & $2298 \mathrm{bp}$ & Lysine & Glutamine \\
\hline AF227982 & 2001, Australia & AF227982 & $2286 \mathrm{bp}$ & Lysine & Glutamine \\
\hline AB046590 & 2001, Japan & AB046590 & $2298 \mathrm{bp}$ & Lysine & Glutamine \\
\hline AF475908 & 2002, China & AF475908 & $2298 \mathrm{bp}$ & Lysine & Glutamine \\
\hline clone 33 & 2002, Germany & AJ297684 & $2298 \mathrm{bp}$ & Lysine & Glutamine \\
\hline SMSC-1P60 & 2003, Malaysia & AF390102 & $2298 \mathrm{bp}$ & Lysine & Glutamine \\
\hline BD-3 & 2004, Bangladesh & AF395114 & $2298 \mathrm{bp}$ & Glutamine & Glutamine \\
\hline TJBD40 & 2004, China & AY846844 & $2298 \mathrm{bp}$ & Lysine & Glutamine \\
\hline AH6 & 2005, China & DQ124935 & $2298 \mathrm{bp}$ & Glutamine & Glutamine \\
\hline TJBD33 & 2005, China & AY843527 & 2298 bp & Lysine & Glutamine \\
\hline SMSC-1P123WT & 2005, Malaysia & DQ217401 & $2298 \mathrm{bp}$ & Lysine & Glutamine \\
\hline CAE26P4 & 2007, Netherlands & D10068 & $2298 \mathrm{bp}$ & Lysine & Glutamine \\
\hline 3711 & 2007, Australia & EF683159 & $2279 \mathrm{bp}$ & Lysine & Glutamine \\
\hline $\mathrm{C} 14$ & 2007, China & EF176599 & $2298 \mathrm{bp}$ & Lysine & Glutamine \\
\hline $01-4201$ & 2007, USA & DQ991394 & $2298 \mathrm{bp}$ & Lysine & Glutamine \\
\hline SDLY08 & 2008, China & FJ172347 & $2298 \mathrm{bp}$ & Lysine & Glutamine \\
\hline CAECA123 & 2008, Japan & D31965 & 2319 bp & Lysine & Glutamine \\
\hline AB119448 & 2009, Japna & AB119448 & $2298 \mathrm{bp}$ & Glutamine & Glutamine \\
\hline 98D02152 & 2010, USA & AF311892 & $2298 \mathrm{bp}$ & Lysine & Glutamine \\
\hline GD-1-12 & 2012, China & JX260426 & 2298 bp & Lysine & Glutamine \\
\hline GD-K-12 & 2013, China & KF224935 & $2298 \mathrm{bp}$ & Glutamine & Glutamine \\
\hline GD-L-12 & 2013, China & KF224936 & $2298 \mathrm{bp}$ & Lysine & Glutamine \\
\hline CIAV89-69 & 2013, Korea & JF507715 & $2298 \mathrm{bp}$ & Lysine & Glutamine \\
\hline CAV-10 & 2014, Argentina & KJ872513 & 2298 bp & Glutamine & Glutamine \\
\hline SC-MS & 2014, China & KM496305 & $2298 \mathrm{bp}$ & Lysine & Glutamine \\
\hline NC001427 & 2015, USA & NC001427 & 2319 bp & Lysine & Aspartate \\
\hline SD1403 & 2016, China & KU221054 & $2298 \mathrm{bp}$ & Glutamine & Glutamine \\
\hline
\end{tabular}

chickens and then inoculated into MDCC-MSB1 cells. This cell line was purchased from ATCC and passaged in our lab for fifteen generations. The blind passage was carried out every three days, until the emergence of cytopathogenic effects (CPE). After CPE, the media of MSB1 cells were collected and repeatedly frozen and thawed three times. The media were then centrifuged, and DNA was extracted using a DNA extraction kit (Tiangen, Beijing, China) from the supernatant. PCR experiments for identification were carried out according to previous studies [8].

2.3. Whole Genome Sequencing. According to published CIAV genome sequences (Table 1), three pairs of primers were designed (Primer Premier 5.0) and three overlapping fragments were amplified, respectively (Table 2). Those fragments were subcloned into PMD-18T vectors (Takara, Dalian, China) and sequenced by Sanger method (Sangon, Shanghai, China). Then, the whole genome sequences of the isolated strains were obtained by assembling those overlapping fragments using Lasergene 7.1 software.

2.4. Sequence Analysis of Isolated Strain. In order to compare the homology of CIAV with different reference strains, the homology of the whole genome and its coding regions (VP1, VP2, and VP3) were analyzed by DNAstar software. Phylogenetic trees were reconstructed on the basis of sequences from the whole genome, VP1, VP2, and VP3 respectively, using MEGA5.1 (NJ) software. The mutation sites of AA from the 139 to 151 region of the VP1 loci in different strains were analyzed statistically.

Nucleotide Sequence Accession Numbers. The GenBank accession number for the sequences of SD1403 was KU221054.

\section{Results}

3.1. The Isolation and Identification of the Virus. $10^{6}$ MDCCMSB1 cells cultured in six-well plates were inoculated with 
TABLE 2: Primers used for genome amplification.

\begin{tabular}{|c|c|c|c|c|}
\hline Primers & Sequence & Location & Temperature & Product length \\
\hline F1 & $5^{\prime}$-GCATTCCGAGTGGTTACTATTCC-3' & \# 1-23 & \multirow{2}{*}{$55^{\circ} \mathrm{C}$} & \multirow{2}{*}{842 bp } \\
\hline $\mathrm{R} 1$ & 5'-CGTCTTGCCATCTTACAGTCTTAT-3' & \# 819-842 & & \\
\hline $\mathrm{F} 2$ & $5^{\prime}$-CGAGTACAGGGTAAGCGAGCTAAA- $3^{\prime}$ & \# 743-767 & \multirow{2}{*}{$55^{\circ} \mathrm{C}$} & \multirow{2}{*}{$990 \mathrm{bp}$} \\
\hline $\mathrm{R} 2$ & 5'-TGCTATTCATGCAGCGGACTT-3' & \# 1712-1732 & & \\
\hline F3 & $5^{\prime}$-ACGAGCAACAGTACCCTGCTAT-3' & \# 1643-1664 & \multirow{2}{*}{$55^{\circ} \mathrm{C}$} & \multirow{2}{*}{802 bp } \\
\hline R3 & 5'-CTGTACATGCTCCACTCGTT-3' & \# 87-151 & & \\
\hline
\end{tabular}

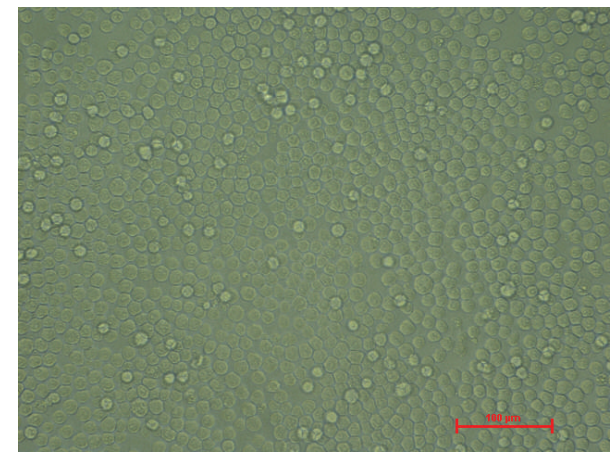

(a)

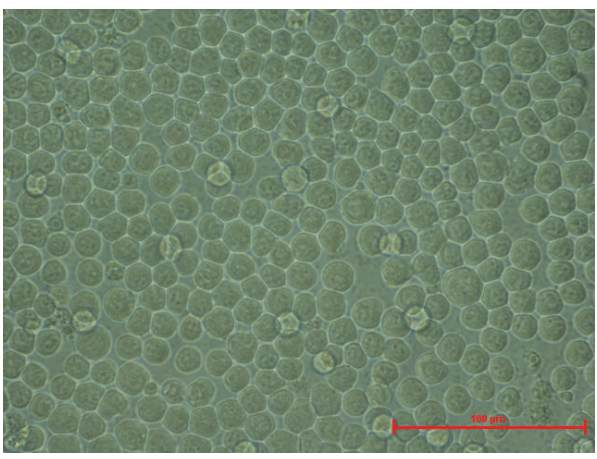

(c)

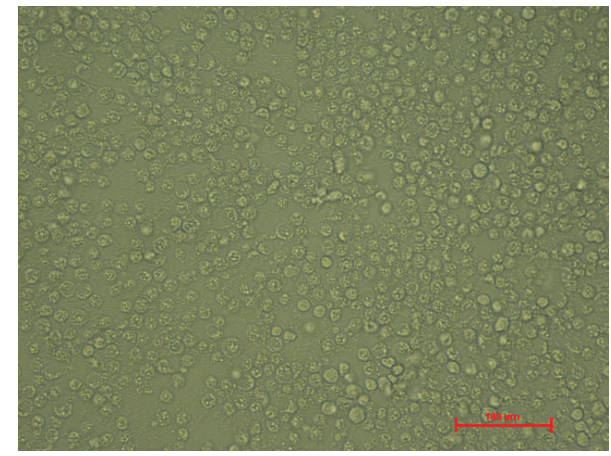

(b)

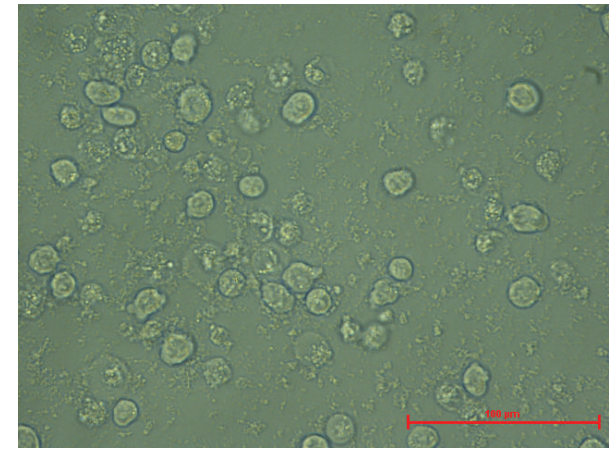

(d)

Figure 1: Cytopathic effect of CIAV isolate on MSB1 cells. (a) Normal MSB1 cells (200x). (b) CPE of MSB1 cells infected with CIAV (200x). (c) Normal MSB1 cells (400x). (d) CPE of MSB1 cells infected with CIAV (400x).

$100 \mu \mathrm{L}$ blood from ten individual SPF chickens which might be infected with CIAV, and, after four blind passages, the cells infected with one blood sample displayed CPEs. Some cells became round and enlarged, and lysis or disintegrating of cells could be observed, with the number of viable cells decreasing, while the morphology of uninoculated MSB1 cells remained normal (Figure 1). A specific PCR band of about $600 \mathrm{bp}$ was amplified from the DNA of MSB1 cells showing $\mathrm{CPE}$ (Figure 2). The result indicated that a CIAV strain was isolated successfully and named as SD1403.

3.2. Genome Sequencing of the Isolate. The full genome of SD1403 strain was 2298 bp, including three ORFs (VP1, VP2, and VP3). VP1, VP2, and VP3 were $1350 \mathrm{bp}, 651 \mathrm{bp}$, and $366 \mathrm{bp}$ in length, respectively. Comparison between the whole genome sequences of the SD1403 strain and 30 other strains was carried out, and the results demonstrated that the homology was $94.8 \%-98.5 \%$. A phylogenic tree was constructed using whole genome sequence of SD1403 showing

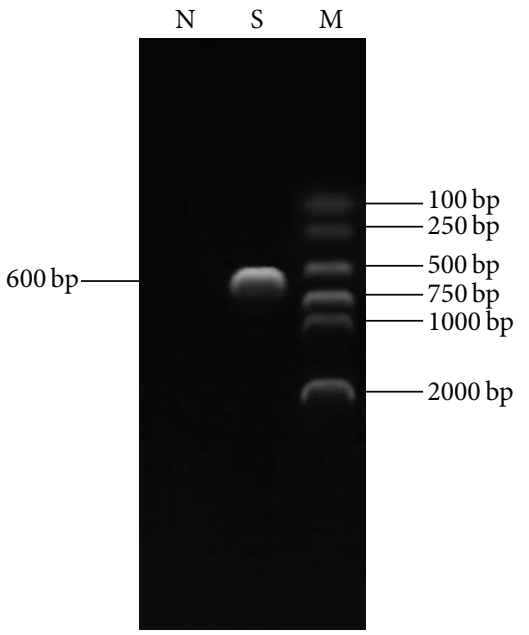

FIGURE 2: Identification by PCR amplification of the CIAV isolates. $\mathrm{N}$ : MSB1 cell without CIAV infection as negative control; S: sample detected in the current study; M: marker. 


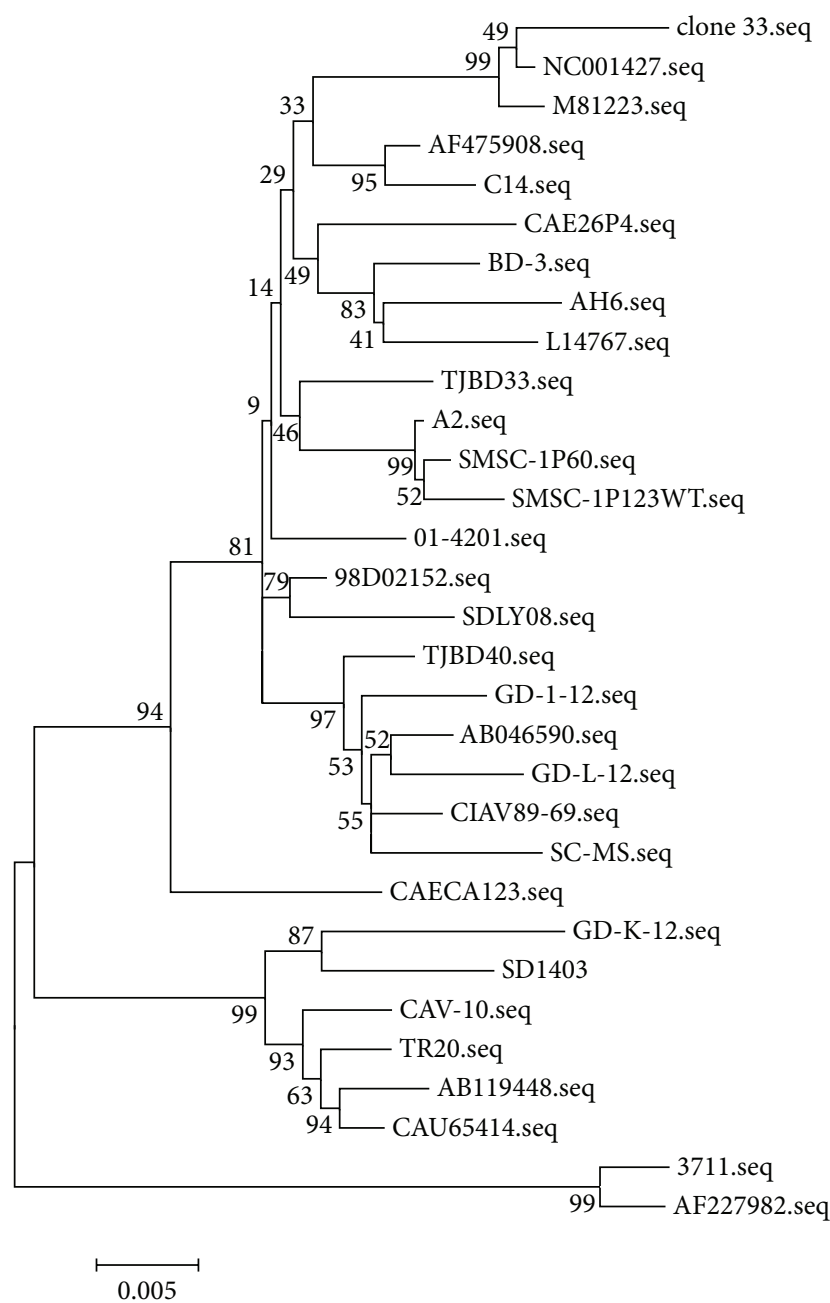

(a)

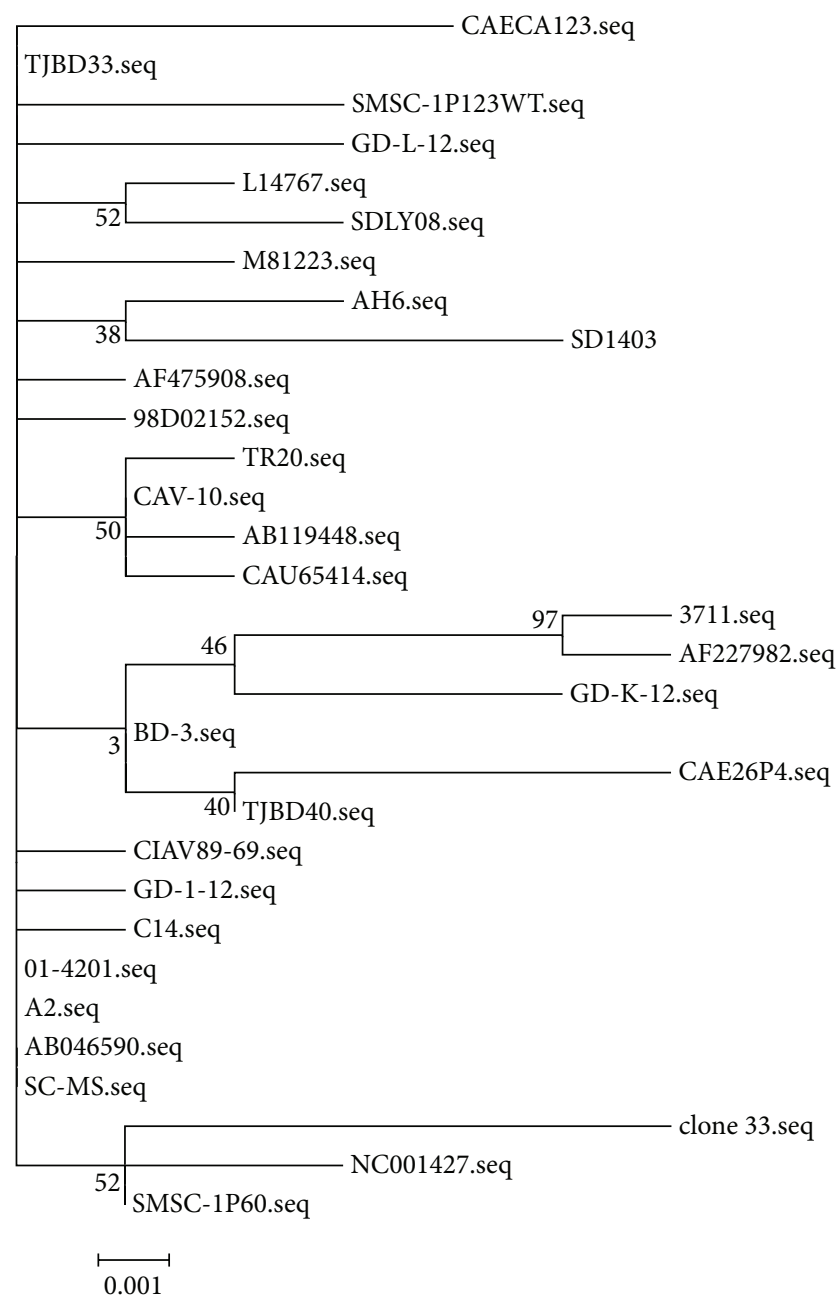

(b)

FIGURE 3: Phylogenetic analysis of different CIAV isolates based on whole genome sequence and VP3 protein. (a) Phylogenetic analysis of CIAV isolates based on whole genome. (b) Phylogenetic analysis of VP3 protein from different CIAV isolates.

that SD1403 was closely related to the GD-K-12 strain, which has low pathogenicity and was isolated in China in 2013 (Figure 3(a)).

Compared with other reference strains, the homology of VP1, VP2, and VP3 from the SD1403 strain was 97.7\%-98.3\%, $98.3 \%-99.2 \%$, and $97.8 \%-99.5 \%$, respectively. It was shown that the sequences of the three genes were highly conserved. VP2 was more conserved than VP1, with the most conserved gene being VP3. The phylogenetic trees were constructed on the basis of VP1, VP2, and VP3 proteins. Phylogenetic tree which was constructed on the basis of VP1 protein was similar to that constructed on the basis of whole genome. It showed that SD1403 and GD-K-12 were in the same clade, indicating that VP1 was more representative between the strains. The SD1403 and GD-K-12 strains were isolated in China and had common characteristics, with AA 139 and 144 of VP1 being glutamine (Table 1). Phylogenetic tree constructed on the basis of VP2 protein was similar to that constructed on the basis of VP3 protein, indicating that both of them were relatively conserved (Figure 3(b)).

\section{Discussion}

A lot of attenuated vaccines for poultry have been confirmed to contain some exogenous viruses. The most reported viruses are Avian leukosis virus (ALV) $[9,10]$ and Reticuloendotheliosis virus (REV) [11-13], with CIAV also being reported [8]. The main reason for this phenomenon is that vaccines made from SPF chicken embryos contaminated by viruses have been used. These viruses (ALV, CIAV, REV, etc.) are mainly transmitted vertically. In China, it has been previously reported that SPF chicken populations were infected with ALV [14]. This is the first report of a CIAV strain isolated and identified in a SPF chicken population in China.

To analyze the genetic variation of this strain and track its possible origin, we sequenced its whole genome. Similar to other CIAV strains, the genomic sequence of SD1403 was highly conserved, and the homology of nucleotide sequence with other reference strains was $94.8 \%-98.5 \%$. From phylogenetic trees constructed on the basis of genomic sequences, the SD1403 and GD-K-1 were closely related, and results were 
very similar to the phylogenetic trees constructed on the basis of VP1, showing that VP1 is more representative between the strains.

It was reported that pathogenicity and infectivity of GDK-12 were weak [3], so we speculated that SD1403 might also be an attenuated strain. Previous studies indicated that if AA 139 and/or AA 144 of VP1 were glutamines, the infectivity and replication were weak. Both AA 139 and 144 of VP1 from SD1403 were glutamines, and these traits were in accordance with GD-K-1 and AH6 strains which were isolated in China. We observed that the SPF chicken population might have been infected with the SD1403 strain for a long time, and the results found that it did not show obvious pathogenicity. It also revealed that SPF chickens raised at the same time did not have pathogenicity, indicating that the pathogenicity and transmissibility of SD1403 strain were weak. According to reports, vaccines contaminated with ALV and REV might cause more serious damage, maybe because ALV and REV have relatively strong pathogenicity [15]. The transmissibility and pathogenicity of SD1403 were weak without showing clinical signs and symptoms. Perhaps this was an important reason why chicken populations were infected with SD1403 latently. Maybe because ALV, REV, and CIAV have different pathogenicity, the avian vaccines contaminated by REV and ALV were more than those by CIAV in the past years.

Although the CIAV infection in SPF chicken was relatively weak, we could not ignore its potential damage. On the one hand, when vaccine contaminated with CIAV is used in a younger chicken population, the pathogenicity of CIAV might be enhanced; on the other hand, if some chicken populations are infected with both ALV and REV, the pathogenicity of CIAV could also be enhanced [16]. Therefore, we must keep detecting CIAV in SPF chicken populations and attenuated vaccines.

\section{Competing Interests}

The authors declare that they have no competing interests.

\section{Authors' Contributions}

Yang Li and Yixin Wang have contributed equally to this work.

\section{References}

[1] J. K. Rosenberger and S. S. Cloud, "The isolation and characterization of chicken anemia agent (CAA) from broilers in the United States," Avian Diseases, vol. 33, no. 4, pp. 707-713, 1989.

[2] S. Natesan, J. M. Kataria, K. Dhama, S. Rahul, and N. Baradhwaj, "Biological and molecular characterization of chicken anaemia virus isolates of Indian origin," Virus Research, vol. 118, no. 1-2, pp. 78-86, 2006.

[3] Y. M. Eltahir, K. Qian, W. Jin, P. Wang, and A. Qin, "Molecular epidemiology of chicken anemia virus in commercial farms in China," Virology Journal, vol. 8, article 145, 2011.

[4] P. Bhatt, S. K. Shukla, M. Mahendran, K. Dhama, M. M. Chawak, and J. M. Kataria, "Prevalence of Chicken Infectious
Anaemia Virus (CIAV) in commercial poultry flocks of Northern India: a serological survey," Transboundary and Emerging Diseases, vol. 58, no. 5, pp. 458-460, 2011.

[5] N. Yuasa, I. Yoshida, and T. Taniguchi, "Isolation of a reticuloendotheliosis virus from chickens inoculated with Marek's disease vaccine," National Institute of Animal Health Quarterly, vol. 16, no. 4, pp. 141-151, 1976.

[6] G. Koch, D. J. van Roozelaar, C. A. J. Verschueren, A. J. van der Eb, and M. H. M. Noteborn, "Immunogenic and protective properties of chicken anaemia virus proteins expressed by baculovirus," Vaccine, vol. 13, no. 8, pp. 763-770, 1995.

[7] R. W. Renshaw, C. Soiné, T. Weinkle et al., "A hypervariable region in VP1 of chicken infectious anemia virus mediates rate of spread and cell tropism in tissue culture," Journal of Virology, vol. 70, no. 12, pp. 8872-8878, 1996.

[8] H. Shao, P. Wang, M. Wu, W. Jin, K. Qian, and A. Qin, "Rapid PCR approach for detecting the contamination of chicken anemia virus in avian live vaccines," China Poultry, vol. 21, article 10, 2012.

[9] A. M. Fadly, R. L. Witter, E. J. Smith et al., "An outbreak of lymphomas in commercial broiler breeder chickens vaccinated with a fowlpox vaccine contaminated with reticuloendotheliosis virus," Avian Pathology, vol. 25, no. 1, pp. 35-47, 1996.

[10] P. Zhao, X. Dong, and Z. Cui, "Isolation, identification, and gp85 characterization of a subgroup A avian leukosis virus from a contaminated live Newcastle Disease virus vaccine, first report in China," Poultry Science, vol. 93, no. 9, pp. 2168-2174, 2014.

[11] A. M. Awad, H. S. A. El-Hamid, A. A. A. Rawash, and H. H. Ibrahim, "Detection of reticuloendotheliosis virus as a contaminant of fowl pox vaccines," Poultry Science, vol. 89, no. 11, pp. 2389-2395, 2010.

[12] S. K. Biswas, C. Jana, K. Chand, W. Rehman, and B. Mondal, "Detection of fowl poxvirus integrated with reticuloendotheliosis virus sequences from an outbreak in backyard chickens in India," Veterinaria Italiana, vol. 47, no. 2, pp. 147-153, 2011.

[13] A. Fadly and M. C. Garcia, "Detection of reticuloendotheliosis virus in live virus vaccines of poultry," Developments in Biologicals, vol. 126, pp. 301-305, 2006.

[14] T. Barbosa, G. Zavala, and S. Cheng, "Molecular characterization of three recombinant isolates of avian leukosis virus obtained from contaminated Marek's disease vaccines," Avian Diseases, vol. 52, no. 2, pp. 245-252, 2008.

[15] K. Wei, Z. Sun, S. Zhu et al., "Probable congenital transmission of reticuloendotheliosis virus caused by vaccination with contaminated vaccines," PLoS ONE, vol. 7, no. 8, Article ID e43422, 2012.

[16] Y. Li, Z. Z. Cui, S. Jiang, and H. Guo, "Synergic inhibitory effect of co-infection of CAV and REV on immune responses to vaccines in SPF chickens," Chinese Journal of Veterinary Science, vol. 28, no. 11, pp. 1243-1246, 2008. 

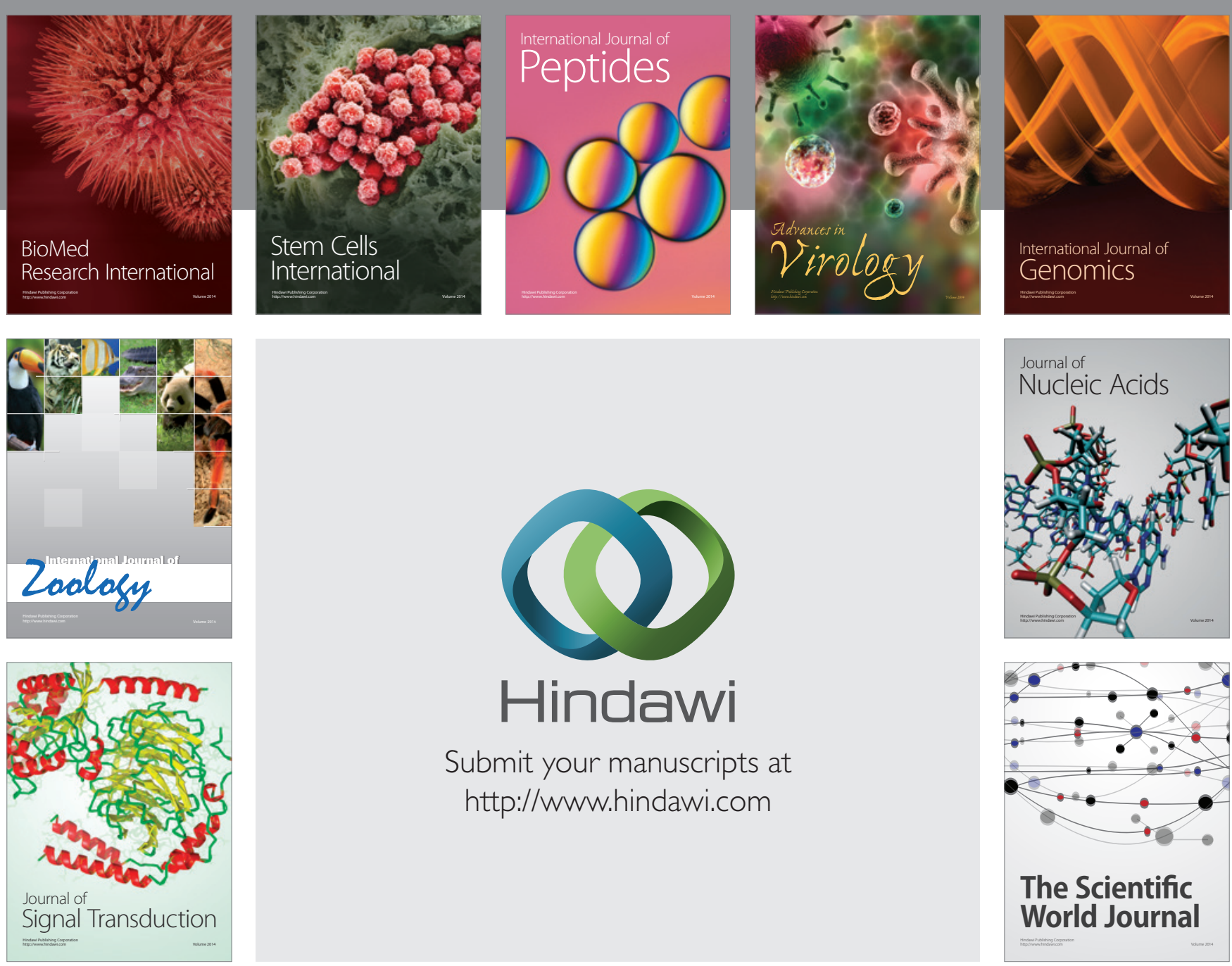

Submit your manuscripts at

http://www.hindawi.com
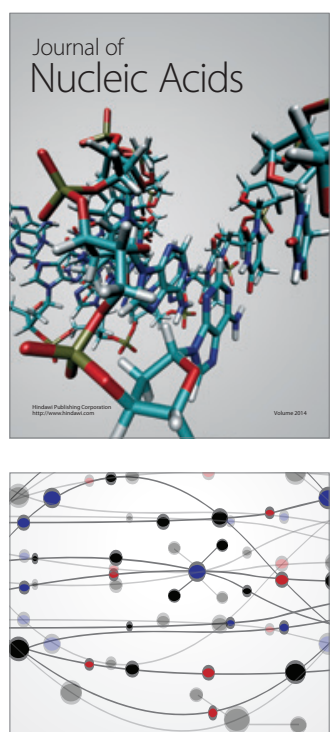

The Scientific World Journal
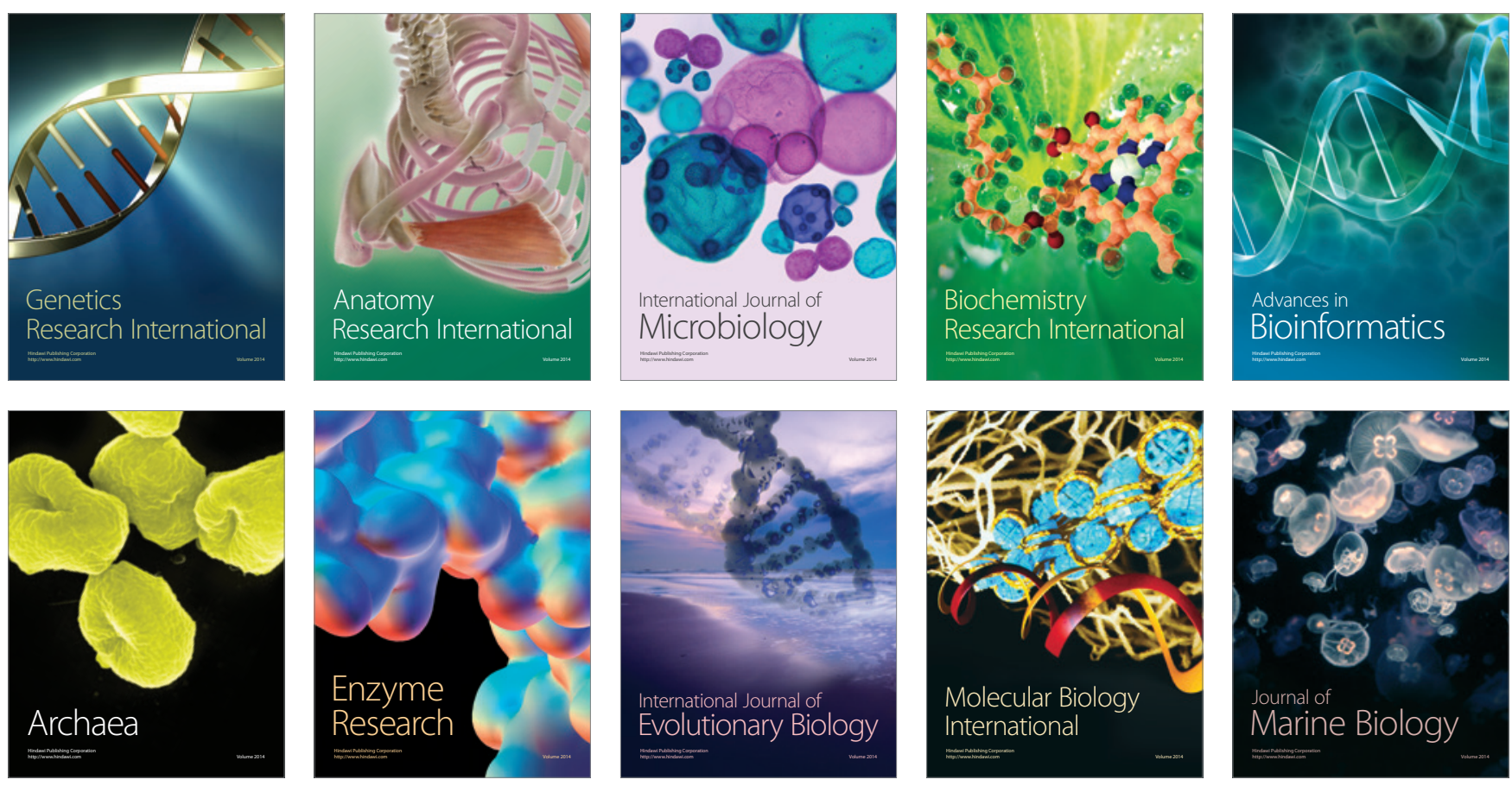